PROCEEDINGS OF THE

AMERICAN MATHEMATICAL SOCIETY

Volume 131, Number 6, Pages 1751-1758

S 0002-9939(03)06927-2

Article electronically published on January 2, 2003

\title{
EXISTENCE OF GIBBS MEASURES FOR COUNTABLE MARKOV SHIFTS
}

\author{
OMRI SARIG
}

(Communicated by Michael Handel)

\begin{abstract}
We prove that a potential with summable variations and finite pressure on a topologically mixing countable Markov shift has a Gibbs measure iff the transition matrix satisfies the big images and preimages property. This strengthens a result of D. Mauldin and M. Urbański (2001) who showed that this condition is sufficient.
\end{abstract}

\section{INTRODUCTION}

Let $S$ be a countable set and $\mathbb{A}=\left(t_{i j}\right)_{S \times S}$ a matrix of zeroes and ones with no columns or rows which are all made of zeroes. Let

$$
X:=\left\{x \in S^{\mathbb{N} \cup\{0\}}: \forall i \quad t_{x_{i} x_{i+1}}=1\right\}
$$

be the corresponding topological Markov shift, equipped with the topology generated by the base of cylinder sets $\left[a_{0}, \ldots, a_{n-1}\right]:=\left\{x \in X: x_{i}=a_{i}\right\}$. Let $T: X \rightarrow X$ be the left shift, $(T x)_{i}=x_{i+1}$. We say that $X$ is topologically mixing if $(X, T)$ is topologically mixing as a topological dynamical system. Let $\phi: X \rightarrow \mathbb{R}$ be some real function. The variations of $\phi$ are defined as

$$
V_{n}(\phi):=\sup \left\{|\phi(x)-\phi(y)|:\left(x_{0}, \ldots, x_{n-1}\right)=\left(y_{0}, \ldots, y_{n-1}\right)\right\} .
$$

A Gibbs measure for $\phi$ is an invariant probability measure $m$ such that for some global constants $P$ and $B$ and every cylinder $\left[a_{0}, \ldots, a_{n-1}\right]$,

$$
\frac{1}{B} \leqslant \frac{m\left[a_{0}, \ldots, a_{n-1}\right]}{e^{\phi_{n}(x)-n P}} \leqslant B \text { for all } x \in\left[a_{0}, \ldots, a_{n-1}\right] .
$$

Here and throughout, $\phi_{n}:=\sum_{k=0}^{n-1} \phi \circ T^{k}$. If $|S|<\infty$ and $\sum_{k \geqslant 1} V_{k}(\phi)<\infty$, then $\phi$ has a Gibbs measure [4, [8], but this may be false if $|S|=\infty$. Indeed, if $|S|=\infty$ the existence of Gibbs measures already implies certain restrictions on the transition matrix $\mathbb{A}$ (theorem 8 in [10]).

Recently, Mauldin and Urbański found sufficient topological conditions for the existence of Gibbs measures ([7, corollary 6.4). In this short note we show that their condition is also necessary. We also show how Mauldin and Urbański's sufficiency result can be derived from the generalized Ruelle's Perron-Frobenius theorem of [11], thus giving a new proof for their result.

Received by the editors October 5, 2001.

2000 Mathematics Subject Classification. Primary 37A99, 37D35; Secondary 37B10.

Key words and phrases. Gibbs measures, countable Markov shifts, thermodynamic formalism. This work is part of a Tel-Aviv University dissertation. 
We need some definitions to be able to state our result. Suppose $(X, T)$ is topologically mixing and that $\phi: X \rightarrow \mathbb{R}$ is some function with $\sum_{k \geqslant 1} V_{k}(\phi)<\infty$. The Gurevich pressure of $\phi$ is defined to be the limit

$$
P_{G}(\phi):=\lim _{n \rightarrow \infty} \frac{1}{n} \log \sum_{T^{n} x=x} e^{\phi_{n}(x)} 1_{[a]}(x)
$$

where $a$ is some element of $S$. It can be shown that this limit exists, is independent of $a$, and that if $\left\|\sum_{T y=x} e^{\phi(y)}\right\|_{\infty}<\infty$, then $P_{G}(\phi)=\sup \left\{h_{\mu}(T)+\int \phi d \mu\right.$ : $\mu$ is a Borel invariant probability s.t. $\left.-\int \phi d \mu<\infty\right\}$ (see [10]).

Finally, denote the transition matrix of $X$ by $\mathbb{A}=\left(t_{i j}\right)_{S \times S}$. The big images and preimages (BIP) property is

$$
\exists b_{1}, \ldots, b_{N} \in S \text { such that } \forall a \in S \exists i, j \text { such that } t_{b_{i} a} t_{a b_{j}}=1 .
$$

This is stronger than the big images property used in [2], and for $X$ topologically mixing, is equivalent to the finite primitivity condition used by Mauldin and Urbański in [7]. The purpose of this short note is to prove:

Theorem 1. If $(X, T)$ is topologically mixing and $\sum_{k \geqslant 1} V_{k}(\phi)<\infty$, then $\phi$ has an invariant Gibbs measure iff $\mathbb{A}$ has the BIP property and $\phi$ has finite Gurevich pressure.

We remark that contrary to most of the results in [10, 11, 12, the condition $\sum_{k \geqslant 1} V_{k}(\phi)<\infty$ cannot be relaxed to $\sum_{k \geqslant 2} V_{k}(\phi)<\infty$, since $V_{1}(\phi)$ must be finite for Theorem 1 to be true.

More results concerning the thermodynamic formalism of countable Markov shifts with the BIP property can be found in section 3, as well as some counterexamples of what might happen in the absence of this property.

The results of this paper can be summarized by the statement that as far as the thermodynamic formalism of D. Ruelle and R. Bowen is concerned, the countable Markov shifts which behave like subshifts of finite type are the countable Markov shifts with the BIP property.

\section{Proof of Theorem 1}

We deduce Theorem 11 from a generalization of Ruelle's Perron-Frobenius theorem from [11] which we now proceed to describe 1 Fix some $a \in S$ and define $\varphi_{a}(x):=1_{[a]}(x) \inf \left\{n \geqslant 1: T^{n}(x) \in[a]\right\}$ (here $\inf \varnothing:=\infty$ and $0 \cdot \infty=0$ ). Set

$$
Z_{n}(\phi, a):=\sum_{T^{n} x=x} e^{\phi_{n}(x)} 1_{[a]}(x) \text { and } Z_{n}^{*}(\phi, a):=\sum_{T^{n} x=x} e^{\phi_{n}(x)} 1_{\left[\varphi_{a}=n\right]}(x) .
$$

Let $\lambda:=\exp \left[P_{G}(\phi)\right]$. We say that $\phi$ is recurrent if for some $a \in S, \sum_{n \geqslant 1} \lambda^{-n} Z_{n}(\phi, a)$ diverges and transient if it converges. We say that $\phi$ is positive recurrent if it is recurrent and $\sum_{n \geqslant 1} n \lambda^{-n} Z_{n}^{*}(\phi, a)<\infty$ and null recurrent if it is recurrent and $\sum_{n \geqslant 1} n \lambda^{-n} Z_{n}^{*}(\phi, a)=\infty$. It is proved in [1] that these definitions do not depend on $a$ and if $\left(L_{\phi} f\right)(x)=\sum_{T y=x} e^{\phi(y)} f(y)$ is Ruelle's operator, then

1 The version which was proved in [12] used stronger continuity assumptions on $\phi$, but the proof given there works verbatim in our context. 
Theorem 2. Suppose $(X, T)$ is topologically mixing and that $\sum_{k \geqslant 2} V_{k}(\phi)<\infty$. If $\phi$ has finite Gurevich pressure, then $\phi$ is recurrent iff there exist $\lambda>0$, a conservative measure $\nu$ finite and positive on cylinders, and a positive continuous function $h$ such that $L_{\phi}^{*} \nu=\lambda \nu$ and $L_{\phi} h=\lambda h$. In this case $\lambda=\exp P_{G}(\phi)$ and there exist $a_{n} \uparrow \infty$ such that for every cylinder $[\underline{a}]$ and $x \in X$

$$
\frac{1}{a_{n}} \sum_{k=1}^{n} \lambda^{-k}\left(L_{\phi}^{k} 1_{[\underline{a}]}\right)(x) \underset{n \rightarrow \infty}{\longrightarrow} h(x) \nu[\underline{a}] .
$$

The sequence $\left\{a_{n}\right\}_{n>0}$ satisfies $a_{n} \sim\left(\int_{[a]} h d \nu\right)^{-1} \sum_{k=1}^{n} \lambda^{-k} Z_{k}(\phi, a)$ for all $a \in S$, and

(1) if $\phi$ is positive recurrent, then $\nu(h)<\infty, \exists c \neq 0$ s.t. $a_{n} \sim$ cn, and $\forall[\underline{a}]$ $\lambda^{-n} L_{\phi}^{n} 1_{[\underline{a}]} \underset{n \rightarrow \infty}{\longrightarrow} h \nu[\underline{a}]$ uniformly on compacts, where $h$ is normalized so that $\nu(h)=1$.

(2) if $\phi$ is null recurrent, then $\nu(h)=\infty, a_{n}=o(n)$, and for every $[\underline{a}]$, $\lambda^{-n} L_{\phi}^{n} 1_{[\underline{a}]}$ tends to zero uniformly on cylinders.

Proof of Theorem 1. We divide the proof into two parts: Necessity and Sufficiency.

Sufficiency. This follows from the work of Mauldin and Urbański [7], but we give a different proof, based on Theorem 2. Assume that $\mathbb{A}$ has the BIP property and that $P_{G}(\phi)<\infty$.

We prove that $\phi$ is positive recurrent. Fix some $a \in S$ and set $\lambda:=e^{P_{G}(\phi)}$. By Theorem 2 positive recurrence is equivalent to the existence of $N_{1} \in \mathbb{N}$, such that

$$
\inf \left\{\lambda^{-n} Z_{n}(\phi, a): n \geqslant N_{1}\right\}>0 \text {. }
$$

This is because (3) implies recurrence and rules out null recurrence, since for every $x \in[a], \lambda^{-n} L_{\phi}^{n} 1_{[a]}(x) \asymp \lambda^{-n} Z_{n}(\phi, a) \neq o(1)$.

Let $\mathcal{W}_{n}:=\left\{\underline{a} \in S^{n}:[\underline{a}] \neq \varnothing\right\}$. For every $\underline{a} \in \mathcal{W}_{n}$, set

$$
\phi_{n}(\underline{a}):=\inf \left\{\phi_{n}(x): x \in[\underline{a}]\right\} \text { and } \phi_{n}[\underline{a}]:=\sup \left\{\phi_{n}(x): x \in[\underline{a}]\right\} .
$$

These are finite, since $V_{1}(\phi)<\infty$. In fact, if $B_{0}:=\exp \sum_{k \geqslant 1} V_{k}(\phi)$, then for all $\underline{w} \in \mathcal{W}_{n},\left|\phi_{n}(\underline{w})-\phi_{n}[\underline{w}]\right| \leqslant \log B_{0}$.

Let $b_{1}, \ldots, b_{N} \in S$ be as in (2). Since $X$ is topologically mixing, there is some $n_{1}$ and admissible words $\underline{w}_{a b_{i}}, \underline{w}_{b_{j} a} \in \mathcal{W}_{n_{1}}$, such that $\left(a, \underline{w}_{a b_{i}}, b_{i}\right)$ and $\left(b_{j}, \underline{w}_{b_{j} a}, a\right)$ are admissible for $i, j=1, \ldots, N$. By (2) for every $\underline{w} \in \mathcal{W}_{n}$ there are $1 \leqslant i, j \leqslant N$ such that $\left(b_{i}, \underline{w}, b_{j}\right)$ is admissible, and so

$$
\left(a, \underline{w}_{a b_{i}}, b_{i}, \underline{w}, b_{j}, \underline{w}_{b_{j} a}, a\right) \in \mathcal{W}_{n+N_{1}+1} \text { where } N_{1}:=2 n_{1}+3
$$

Set $C:=\min \left\{e^{\phi_{n_{1}+2}\left(a, \underline{w}_{a b_{i}}, b_{i}\right)} \cdot e^{\phi_{n_{1}+1}\left(b_{j}, \underline{w}_{b_{j} a}\right)}: i, j=1, \ldots, N\right\}$. If $C_{1}:=\frac{C}{\lambda^{N_{1}}}$, then

$$
\lambda^{-\left(n+N_{1}\right)} Z_{n+N_{1}}(\phi, a) \geqslant C_{1} \lambda^{-n} \sum_{\underline{w} \in \mathcal{W}_{n}} e^{\phi_{n}(\underline{w})} \geqslant \frac{C_{1}}{B_{0}} \lambda^{-n} \sum_{\underline{w} \in \mathcal{W}_{n}} e^{\phi_{n}[\underline{w}]} .
$$

We claim that the last expression is bounded from below. Indeed, assume by way of contradiction that $\exists n_{0}$ such that $\lambda^{-n_{0}} \sum_{\underline{w} \in \mathcal{W}_{n_{0}}} e^{\phi_{n_{0}}[\underline{w}]}<\frac{1}{2}$. Then for all $k$

$$
\lambda^{-k n_{0}} Z_{k n_{0}}(\phi, a) \leqslant\left(\lambda^{-n_{0}} \sum_{\underline{w} \in \mathcal{W}_{n_{0}}} e^{\phi_{n_{0}}[\underline{w}]}\right)^{k}<\frac{1}{2^{k}} .
$$


This, however, is impossible since $\lambda^{-k n} Z_{k n}(\phi, a)$ cannot decay exponentially fast. This proves (3), and the positive recurrence of $\phi$ follows.

Let $\nu$ and $h$ be the eigenmeasure and eigenfunction given by Theorem 2 normalized so that $\int h d \nu=1$, and set $d m:=h d \nu$. We prove that $\sup h<\infty$ and $\inf h>0$. Fix some $a \in S$. By Theorem 2 and (4),

$$
\begin{aligned}
h(x) & =\frac{1}{\nu[a]} \lim _{n \rightarrow \infty} \lambda^{-n} L_{\phi}^{n} 1_{[a]}(x) \\
& \leqslant \frac{1}{\nu[a]} \sup _{n \geqslant 1}\left\{\lambda^{-n} \sum_{\underline{w} \in \mathcal{W}_{n}} e^{\phi_{n}[\underline{w}]}\right\} \leqslant \frac{B_{0}}{C_{1} \nu[a]} \sup _{n \geqslant 1} \lambda^{-n} Z_{n}(\phi, a) .
\end{aligned}
$$

The last supremum in the above equation is bounded by $2 B_{0}$, because if $\exists k_{0}$ such that $\lambda^{-k_{0}} Z_{k_{0}}(\phi, a) \geqslant 2 B_{0}$, then for every $n, \lambda^{-k_{0} n} Z_{k_{0} n}(\phi, a) \geqslant B_{0}^{-n}\left(\lambda^{-k_{0}} Z_{k_{0}}(\phi, a)\right)^{n}$ $\geqslant 2^{n}$ which is impossible by the definition of $\lambda$. Therefore $\sup h<\infty$. To see that $\inf h>0$, note first that $h$ is bounded from below on partition sets, because $h(x)=\frac{1}{\nu[a]} \lim _{n \rightarrow \infty} \lambda^{-n} \sum_{T^{n} y=x} e^{\phi_{n}(y)} 1_{[a]}(y)$ and $\sup _{n} V_{1}\left(\phi_{n}\right) \leqslant \sum_{k \geqslant 1} V_{k}(\phi)<\infty$. Next, note that the BIP property implies that for every $x \in X, \exists 1 \leqslant i \leqslant N$ such that $\left(b_{i}, x\right)$ is admissible. Therefore, $h(x)=\lambda^{-1}\left(L_{\phi} h\right)(x) \geqslant \lambda^{-1} e^{\phi\left(b_{i} x\right)} h\left(b_{i} x\right)$. The last quantity is bounded from below, since there are only finitely many $b_{i}$ 's. Thus inf $h>0$.

We now show that $h d \nu$ is an invariant Gibbs measure, proving the sufficiency part of the theorem. Invariance is clear, so we focus on (11). Fix some $H$ such that $\frac{1}{H}<h(x)<H$ for all $x$. Fix some $\underline{a} \in \mathcal{W}_{n}$ and $x \in[\underline{a}]$. Using the notation $a=M^{ \pm} b$ to denote the double inequalities $M^{-1} b \leqslant a \leqslant M b$, we have

$$
\begin{aligned}
m[\underline{a}] & =\int \lambda^{-n} L_{\phi}^{n}\left(h 1_{[\underline{a}]}\right) d \nu=H^{ \pm 1} \int \lambda^{-n} L_{\phi}^{n} 1_{[\underline{a}]} d \nu \\
& =\left(H B_{0}\right)^{ \pm 1} e^{\phi_{n}(\underline{a})-n \log \lambda} \nu\left(T^{n}[\underline{a}]\right)=\left(H^{2} B_{0}\right)^{ \pm 1} e^{\phi_{n}(\underline{a})-n \log \lambda} m\left(T^{n}[\underline{a}]\right) .
\end{aligned}
$$

It is, therefore, enough to show that $m\left(T^{n}[\underline{a}]\right)$ is bounded away from zero and infinity. Boundness from above is clear, since $m(X)=1$. Boundness from below follows from $m\left(T^{n}[\underline{a}]\right) \geqslant \min \left\{m\left[b_{i}\right]: i=1, \ldots, N\right\}>0$.

Necessity. Assume now that there is an invariant Gibbs measure $m$. If $B$ and $P$ are as in (1), then

$$
\begin{aligned}
Z_{n}(\phi, a) & \leqslant \sum_{\left(a, \xi_{1}, \ldots, \xi_{n-1}, a\right) \in \mathcal{W}_{n+1}} e^{\phi_{n}\left[a, \xi_{1}, \ldots, \xi_{n-1}, a\right]} \\
& \leqslant B e^{n P} \sum_{\left(a, \xi_{1}, \ldots, \xi_{n-1}, a\right) \in \mathcal{W}_{n+1}} m\left[a, \xi_{1}, \ldots, \xi_{n-1}, a\right] \leqslant B e^{n P} .
\end{aligned}
$$

Consequently, $P_{G}(\phi) \leqslant P<\infty$. (Actually, $P_{G}(\phi)=P$, but we do not need this.)

Next, we show that $\mathbb{A}$ satisfies BIP. By (1), and since $V_{1}(\phi)<\infty$, there is some global constant $C$, such that for all $p, q \in S$, such that $[p, q] \neq \varnothing$,

$$
m[p, q]=C^{ \pm 1} e^{\phi(p)} \cdot e^{\phi(q)} .
$$


Summing over all possibilities, first for $p$ (fixing $q$ ) and then for $q$ (fixing $p$ ), we have

$$
\begin{aligned}
m\left(T^{-1}[q]\right) & =C^{ \pm 1} e^{\phi(q)} \sum_{p:[p, q] \neq \varnothing} e^{\phi(p)}, \\
m[p] & =C^{ \pm 1} e^{\phi(p)} \sum_{q:[p, q] \neq \varnothing} e^{\phi(q)}
\end{aligned}
$$

whence, since $m\left(T^{-1}[q]\right)=m[q]=B^{ \pm 1} e^{-P} e^{\phi(q)}$ and $m[p]=B^{ \pm 1} e^{-P} e^{\phi(p)}$,

$$
\inf \left\{\sum_{a:[a, q] \neq \varnothing} e^{\phi(a)}, \sum_{a:[p, a] \neq \varnothing} e^{\phi(a)}: p, q \in S\right\}>0 .
$$

Inducing an order on $S$, we assume without loss of generality that $S=\mathbb{N}$. Assume by way of contradiction that BIP fails. Then for every $k$, either $\exists q_{k} \in S$, such that $\left\{a:\left[a, q_{k}\right] \neq \varnothing\right\} \subseteq\{a: a>k\}$ or $\exists p_{k} \in S$, such that $\left\{a:\left[p_{k}, a\right] \neq \varnothing\right\} \subseteq\{a: a>k\}$. This means that the infimum in (5I) is equal to zero, since

$$
\sum_{a \in S} e^{\phi(a)} \leqslant B e^{P} \sum_{a \in S} m[a]<\infty .
$$

This contradiction proves the BIP property.

\section{More on Countable Markov Shifts with the BIP Property}

Throughout this section let $(X, T)$ be a topologically mixing countable Markov shift, and $\phi: X \rightarrow \mathbb{R}$ be some function with finite Gurevich pressure such that $\sum_{n \geqslant 1} V_{n}(\phi)<\infty$. The following results are direct consequences of Theorem 1 or its proof. They show that if the BIP property holds, then the behaviour of $(X, \phi)$ from the point of view of the thermodynamic formalism is similar to the behaviour in the case of subshifts of finite type.

Corollary 1. If the BIP property holds, then $P_{G}(\phi)=\lim _{n \rightarrow \infty} \frac{1}{n} \log \sum_{T^{n} x=x} e^{\phi_{n}(x)}$.

Proof. It is always true that $P_{G}(\phi) \leqslant \lim _{n \rightarrow \infty} \frac{1}{n} \log \sum_{T^{n} x=x} e^{\phi_{n}(x)}$. The other inequality follows from (4).

Remark 1. Without the BIP property, the last proposition is wrong: there exist topologically mixing countable Markov shifts $X$ with $\phi: X \rightarrow \mathbb{R}$ with finite Gurevich pressure such that $\sum_{k \geqslant 1} V_{k}(\phi)<\infty$, for which $P_{G}(\phi)<\lim _{n \rightarrow \infty} \frac{1}{n} \log \sum_{T^{n} x=x} e^{\phi_{n}(x)}$.

Proof. Examples can be found for $\phi \equiv 0$ in [9]. We mention just one: let $S=\mathbb{Z}$ and $\mathbb{A}=\left(t_{i j}\right)_{\mathbb{Z} \times \mathbb{Z}}$ where $t_{i j}=1$ if $|i-j| \leqslant 1$ and $t_{i j}=0$ otherwise. If $\phi \equiv 0$, then $P_{G}(\phi)<\infty$, despite the fact that there are infinitely many periodic points of order $n$ for every $n$.

Corollary 2. If the BIP property holds, then $\phi$ is positive recurrent and if $L_{\phi}$ is Ruelle's operator given by $\left(L_{\phi} f\right)(x)=\sum_{T y=x} e^{\phi(y)} f(y)$, then:

(1) there exist $\lambda>0, h>0$ positive continuous, and a conservative Borel measure $\nu$ which is finite and positive on cylinders, such that $L_{\phi} h=\lambda h$ and $L_{\phi}^{*} \nu=\lambda \nu$, and $\int h d \nu<\infty$;

(2) $h$ is bounded away from zero and infinity and $\nu(X)<\infty$. 
Proof. This was proved in the sufficiency part of the proof of Theorem 1 (see also [7]).

Remark 2. If we relax our assumptions, Corollary 2 may be false:

(1) part (1) fails for potentials which are not positive recurrent.

(2) part (2) might fail in the absence of the BIP property.

(3) part (2) might even fail in the presence of the BIP property, if we relax the condition $\sum_{k \geqslant 1} V_{k}(\phi)<\infty$ to $\sum_{k \geqslant 2} V_{k}(\phi)<\infty$ (i.e. allow $V_{1}(\phi)=\infty$ ).

Proof. The first statement follows from Theorem 2 For the second statement, see example 2 in [10. For the last statement consider some topologically mixing $(X, T)$ with the BIP property and some $\phi: X \rightarrow \mathbb{R}$ with finite pressure such that $\sum_{k \geqslant 1} V_{k}(\phi)<\infty$. Let $h$ and $\nu$ be the eigenvectors of $L_{\phi}$ given by Corollary 2 , and choose some $f: X \rightarrow \mathbb{R}_{+}$such that $\sum e^{f(a)} \nu[a]=\infty$. The potential $\psi=\phi+f-f \circ T$ has the same pressure as $\phi$ and satisfies $\sum_{k \geqslant 2} V_{k}(\psi)=\infty$, but the eigenvectors of $L_{\psi}$ are $e^{-f} h$ and $e^{f} d \nu$, so part (2) is false for $\psi$.

Fix some $\theta \in(0,1)$ and define a metric on $X$ by $d(x, y)=\theta^{\min \left\{i: x_{i} \neq y_{i}\right\}}$. Let $\beta$ be the smallest partition with the property that the $\sigma$-algebra generated by $\beta$ contains $\{T[a]: a \in S\}$, and set

$$
D_{\beta} f:=\sup _{B \in \beta} \sup _{x, y \in B, x \neq y} \frac{|f(x)-f(y)|}{d(x, y)} .
$$

Set $\mathcal{L}:=\left\{f: X \rightarrow \mathbb{C}:\|f\|_{\mathcal{L}}:=\|f\|_{\infty}+D_{\beta} f<\infty\right\}$.

Corollary 3. Suppose the BIP property holds and $\exists A>0$ such that $V_{k}(\phi)<A \theta^{n}$ for all $n \geqslant 1$. If $h, \nu$ and $\lambda$ are as in Corollary 2, then $\exists K>0$ and $r \in(0,1)$ such that for all $f \in \mathcal{L},\left\|\lambda^{-n} L_{\phi}^{n} f-h \int f d \nu\right\|_{\mathcal{L}} \leqslant K r^{n}\|f\|_{\mathcal{L}}$.

Proof. This follows from the results in section 2 in [3] (see theorem 4.7.7 in [1]).

Remark 3. Without the BIP property, the last corollary is false.

Proof. Example 1 in [10].

Our last corollary says that phase transitions of the type one expects for countable Markov shifts (see [12, 13]) do not occur. Let $\operatorname{Dir}(\phi)$ be the collection of all $\psi: X \rightarrow \mathbb{R}$ such that there exists $C>0, r \in(0,1)$ and $\epsilon>0$ such that

$$
\forall n \geqslant 1, V_{n}(\psi)<C_{\psi} r^{n} \text { and } \forall|t|<\epsilon, P_{G}(\phi+t \psi)<\infty .
$$

Corollary 4. If the BIP property holds and $\exists A>0, \theta \in(0,1)$ such that $V_{n}(\phi)<$ $A \theta^{n}$ for all $n \geqslant 1$, then for every $\psi \in \operatorname{Dir}(\phi)$ there exists $\epsilon_{0}>0$ such that $\phi+t \psi$ is positive recurrent for all $|t|<\epsilon_{0}$ and for which $t \mapsto P_{G}(\phi+t \psi)$ is real analytic in $\left(-\epsilon_{0}, \epsilon_{0}\right)$.

Proof. We use the machinary of [12, which we now proceed to describe. Fix some state $a \in S$. Set $\bar{S}:=\left\{[\underline{a}]: a_{i}=a\right.$ iff $\left.i=0 ;[\underline{a}, a] \neq \varnothing\right\}, \bar{X}:=\bar{S}^{\mathbb{N} \cup\{0\}}$ and let $\bar{T}: \bar{X} \rightarrow \bar{X}$ be the left shift. Define $\varphi_{a}(x):=1_{[a]}(x) \inf \left\{n \geqslant 1: T^{n} x \in[a]\right\}$ where $\inf \varnothing:=\infty$ and define

$$
\bar{\phi}:=\left(\sum_{k=0}^{\varphi_{a}-1} \phi \circ T^{k}\right) \circ \pi
$$


where $\pi: \bar{X} \rightarrow[a]$ is given by $\pi\left(\left[\underline{a}_{0}\right],\left[\underline{a}_{1}\right], \ldots\right):=\left(\underline{a}_{0}, \underline{a}_{1}, \ldots\right)$. The pair $(\bar{X}, \bar{\phi})$ is called the induced system and $\bar{\phi}$ is called the induced potential (on $[a]$ ). The $a$-discriminant of $\phi$ is defined to be

$$
\Delta_{a}[\phi]:=\sup \left\{P_{G}(\overline{\phi+p}): p \in \mathbb{R} \text { s.t. } P_{G}(\overline{\phi+p}) \text { is finite }\right\} .
$$

It is proved in [12] that if $\Delta_{a}[\phi]>0$, then $\phi$ is positive recurrent, if $\Delta_{a}[\phi]=0$, then $\phi$ is either positive recurrent or null recurrent, and that if $\Delta_{a}[\phi]<0$, then $\phi$ is transient. It is also proved there that if $\Delta_{a}[\phi]>0$, then for every $\psi \in \operatorname{Dir}(\phi)$, $\exists \epsilon_{0}$ such that $\phi+t \psi$ is positive recurrent for all $|t|<\epsilon_{0}$ and that $t \mapsto P_{G}(\phi+t \psi)$ is real analytic in $\left(-\epsilon_{0}, \epsilon_{0}\right)$. It is therefore enough to prove that $\Delta_{a}[\phi]>0$, or in the terminology of [12, that $\phi$ is strongly positive recurrent.

We actually show that $\Delta_{a}[\phi]=+\infty$. It is easy to verify that if we induce on $a$, then $\overline{\phi+p+t 1_{[a]}}=\overline{\phi+p}+t$, whence $P_{G}\left(\overline{\phi+p+t 1_{[a]}}\right)=P_{G}(\overline{\phi+p})+t$. It follows from this that

$$
\Delta_{a}\left[\phi+t 1_{[a]}\right]=\Delta_{a}[\phi]+t .
$$

If $\Delta_{a}[\phi]<\infty$, then for some $t, \Delta_{a}\left[\phi+t 1_{[a]}\right]<0$, which by the previous remarks means that $\phi+t 1_{[a]}$ is transient. However, $P_{G}\left(\phi+t 1_{[a]}\right) \leqslant P_{G}(\phi)+|t|<\infty$ and $\sum_{n \geqslant 1} V_{n}\left(\phi+t 1_{[a]}\right)=\sum_{n \geqslant 1} V_{n}(\phi)<\infty$ so by Corollary $2, \phi+t 1_{[a]}$ is positive recurrent and we arrive at a contradiction.

For examples of the phase transition phenomena which may appear in the absence of the BIP property, we refer the reader to [12, 13].

We finish with some words of caution: invariant Gibbs measures for countable Markov shifts with the BIP property are not necessarily equilibrium measures in the strict sense of [4, 8]. For an example of what might go wrong consider the shift $X=\mathbb{N}^{\mathbb{N}}$ together with the potential $\phi(x)=\log p_{x_{0}}$ where $\left\{p_{n}\right\}$ is a sequence of positive numbers such that $\sum p_{n}=1$ and $p_{n} \propto 1 / 2 n(\log 2 n)^{2}$. The Gibbs measure for $\phi$ is the Bernoulli measure $m\left[a_{0}, \ldots, a_{n-1}\right]=\prod_{i=0}^{n-1} p_{a_{i}}$. For this measure, $h_{m}(T)=+\infty$ and $\int \phi d m=-\infty$ so the sum $h_{m}(T)+\int \phi d m$ is meaningless. For a discussion of the weaker senses in which $m$ may be considered an equilibrium measure, see [5] and [10].

\section{REFERENCES}

[1] Aaronson, J.: An introduction to infinite Ergodic Theory, Math. Surv. and Mono. 50 (1997), AMS. MR 99d:28025

[2] Aaronson, J., Denker, M., Urbaǹski: Ergodic theory of Markov fibered systems and parabolic rational maps. Trans. Am. Math. Soc. 337 (1993), 495-548. MR 94g:58116

[3] Aaronson, J., Denker, M.: Local limit theorems for partial sums of stationary sequences generated by Gibbs-Markov maps. Stoch. Dyn. 1 (2001), no. 2, 193-237. MR 2002h:37014

[4] Bowen, R.: Equilibrium states and the theory of Anosov diffeomorphisms. Lect. Notes in Math. 470, Springer Verlag (1975). MR 56:1364

[5] Buzzi, J., Sarig, O: Uniqueness of equilibrium measures for countable Markov shifts and multi-dimensional piecewise expanding maps. To appear in Erg. Thy. Dynam. Syst.

[6] Gurevic, B.M.: Topological entropy for denumerable Markov chains. Dokl. Akad. Nauk. SSSR 187 (1969); English Transl. in Soviet Math. Dokl. 10 (1969), 911-915. MR 41:7767

[7] Mauldin, R.D., Urbański, M.: Gibbs states on the symbolic space over an infinite alphabet. Israel J. Maths. 125 (2001), 93-130. MR 2002k:37048

[8] Ruelle, D.: Thermodynamic formalism. Encyclopedia of Mathematics and its Applications 5, Addison-Wesley (1978). MR 80g:82017

[9] Salama, I.: On the recurrence of countable topological Markov chains. Pacific J. Math. 134 (1988), 325-341. Errata Pac. J. Math. 140 (1989), 397. MR 90k:54055 
[10] Sarig, O.: Thermodynamic Formalism for Countable Markov Shifts. Ergod. Th. Dynam. Sys. 19 (1999), 1565-1593. MR 2000m:37009

[11] Sarig, O.: Thermodynamic formalism for null recurrent potentials. Israel J. Math. 121 (2001), 285-311. MR 2001m:37059

[12] Sarig, O.: Phase transitions for countable Markov shifts. Commun. Math. Phys. 217 (2001), 555-577. MR 2002b:37040

[13] Sarig, O.: On an example with topological pressure which is not analytic. C.R. Acad. Sci. Serie I: Math. 330 (2000), 311-315. MR 2000m:37020

Mathematics Institute, University of Warwick, Coventry CV4 7AL, England

E-mail address: sarig@maths.warwick.ac.uk 